\title{
A Novel GUI-Based Image Reconstruction Algorithm of EIT Imaging Technique
}

\author{
Ramesh Kumar, GEC Ajmer, India \\ iD https://orcid.org/0000-0001-9822-8246 \\ Shashank Tripathi, GEC Gaya, India
}

\begin{abstract}
Electrical impedance tomography (EIT) is a non-invasive technique that is used to estimate the electrical properties of a medical or non-medical object through the boundary data of the object. It used to achieve functional imaging of different objects by measuring electrical conductivity and impedance parameters. In this paper, a novel image reconstruction algorithm is presented, which is based on graphical user interface (GUI) developed on MATLAB software platform. EIT imaging algorithm consists of a forward problem and an inverse problem. The forward problem is formulated with the conductance matrix, and a non-iterative inverse method is used to estimate the conductivity distribution. Image display and data analysis are implemented and controlled directly in the GUI. The numerical simulations and phantom experiments have been carried out to evaluate the performance of the proposed algorithm and other previous research data through quantitative parameters. The obtained result shows satisfactory and comparable results to other EIT imaging algorithm.
\end{abstract}

\section{KEYWORDS}

Bio-Impedance, Electrical Impedance Tomography, Finite Element Method, Graphical Interface Unit, Image Reconstruction Algorithm, Jacobean Method, Non-Invasive Technique, Phantom

\section{INTRODUCTION}

Most of the medical imaging techniques, such as ultrasonography, X-ray, CT scan, and ultrasound echo radiography have built up a remarkable achievement in medical applications. Much interest has also been shown in imaging using a low electric current with high-frequency and low frequency, which is called Bio-impedance tomography technique (Holder, 2005). The primary goal of Bio-impedance tomography technique is to construct the image within the object to extract different information. Ultrasonography provides the variation in the mechanical property, X-ray shows the variation in electron density of the objects. However, the electrical impedance tomography shows the variation of conductivity, permittivity and electrical property (Kumar et al., 2008b).

Many non-linear and linear algorithms are used for reconstruction of imaging in the medical and industrial field (Mengxing et al., 1998), such as regularization methods, sensitivity matrix method, equipotential back projection, and Newton-Rapson method, etc. The Newton-Rapson method is applicable for non-linear applications. The computation time under this method is higher as compared to linear techniques due to involvement of higher order equation magnitude (Borsic \& Bayford, 2010). Above shown all technique, as provides realistic solutions and require simplifying hypotheses about the problem. All the techniques discussed earlier are having capacity to provide real time solution for the problem by simplifying hypothesis. Finite Element Method (FEM) converts continuous system

\section{DOI: 10.4018/IJCINI.20210701.oa3}

This article, published as an Open Access article on April 23rd, 2021 in the gold Open Access journal, the International Journal of Cognitive Informatics and Natural Intelligence (converted to gold Open Access January 1st, 2021), is distributed under the terms of the Creative Commons Attribution License (http://creativecommons.org/licenses/by/4.0/) which permits unrestricted use, distribution, and production in any medium, provided the author of the original work and original publication source are properly credited. 
solution into approximated discrete system consists of meshes, elements and nodes in finite number (Kilic et al., 1998). Here, we use a finite element model to simulate the impedance distribution field in image tomography (Seok, 2014). The tomography study aims to rebuild conductive platform over electrical distribution through the boundary measured data within the body according to electrical conductivity distribution function (Soni, 2006). From the previous research is found that the finite element method is most suitable for this type of problems of the inhomogeneous field with arbitrary geometry. We employ a finite element model to express the impedance distribution of the object or body field (Olmos, 2012).

The paper consists of various sections for showing progress of work step by step. The EIT methodology has been discussed in section 3 with explanation of hardware and software part of EIT. The method that includes data acquisition has been discussed in section 4 . The algorithm for reconstruction of image has been proposed in Section 5, which consist of electrode model, forward problem, inverse problem and mathematical methods. In the section 6, the Experimental work has been discussed. The validation of the proposed algorithm through the phantom and result has been discussed in section 7 . In section 8 , the conclusion of the work has been discussed.

\section{BACKGROUND}

Many non-linear and linear algorithms are used for reconstruction of imaging in the medical and industrial field (Mengxing et al., 1998), such as regularization methods, sensitivity matrix method, equipotential back projection, and Newton-Rapson method, etc. The Newton-Rapson method is applicable for non-linear applications. The computation time under this method is higher as compared to linear techniques due to involvement of higher order equation magnitude (Borsic \& Bayford, 2010). Above shown all technique, as provides realistic solutions and require simplifying hypotheses about the problem. All the techniques discussed earlier are having capacity to provide real time solution for the problem by simplifying hypothesis. Finite Element Method (FEM) converts continuous system solution into approximated discrete system consists of meshes, elements and nodes in finite number (Kilic et al., 1998). Here, we use a finite element model to simulate the impedance distribution field in image tomography (Seok, 2014). The tomography study aims to establish the distributed conductivity environment with the help of electrical conductivity distribution function using data measured over the boundary within the body (Soni, 2006). From the previous research is found that the finite element method is most suitable for this type of problems of the inhomogeneous field with arbitrary geometry. A finite element model has been employed in the article to express the electrical impedance distribution for the object or body field (Olmos, 2012).

\section{THE PROPOSED TECHNIQUE}

EIT is a technique of imaging that based on the inside impedance (conductivity) distribution of the object, by calculating all voltage measurements from the surface of the object. These voltage measurements are excited by a low amplitude current pulse that flows through the object (Holder, 2005). EIT is founded on the fact that all interior areas of the object under analysis have different conductivity, which is a current pulse flow changes its direction. So this effect leads to boundary voltage changes and can be detected. Electrical impedance tomography technique has become a speedily growing area of research, which is involved many researchers all our worlds. Currently, the main areas of EIT are processed industries, biomedical diagnosis, nondestructive testing, and geophysics (Hinz et al., 2005).

Combination of hardware that are constituted by current sources, multiplexer module, controller unit and serial communicating with the PC is the first part of EIT system (Khalighi, 2012). The first part of EIT System acquires Electrical Signal through array of electrodes, EIT-sensors and analog and digital circuit and feed to the PC. The combination of all elements is shown in figure 1. Analog 


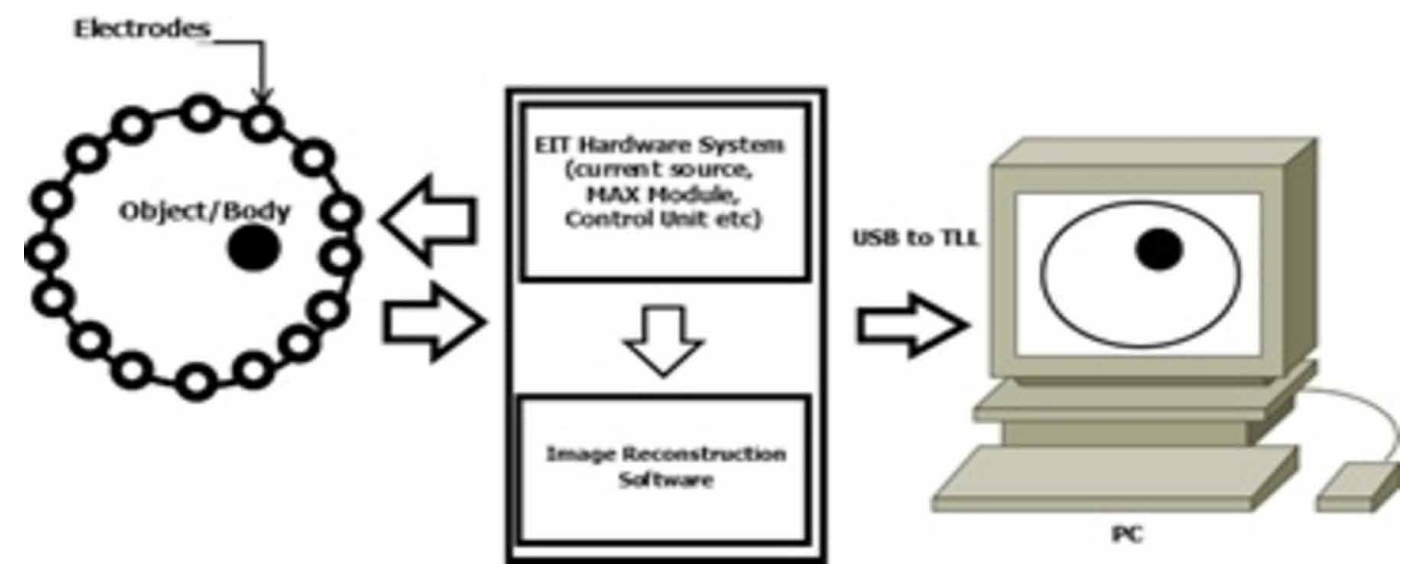

circuitry part of EIT system include data acquisition and source of current. Whereas, programmable microcontroller unit is represented as digital part of EIT system. An image reconstruction algorithm is other significant part of the system. Various linear image reconstruction algorithms have been proposed by researchers including Newton-Rap Son Method, equipotential back projection, Finite Element Method, Tikhonov regularization methods, etc. (Kilic et al., 1998). Here, we use a finite element method to simulate the impedance-based image for a forward solution made of a limited number of nodes and elements, and Jacobean matrix used in inverse problem of the image.

The implemented EIT structure has been illustrated in figure 2 with proper arrangement of current sources, multiplexer module, controller unit and serial communicating with the PC. Low amplitude signal of sinusoidal nature is originated from current source. The voltage signal need to convert into current sinusoidal signal as per desire and requirement of application. The switching of the multiplexer (ADG506) is controlled by control unit (ATMEGA16) (Khalighi, 2012). The multiplexer is most important for EIT method. By implementing the system will be four multiplexers is used. Two multiplexers for data receiving from an object or phantom. Moreover, other two used for current switching to the object. Then, we used a serial communication device (USB to TTL) for data transferring from the control unit to the PC and after getting data from the object, simulation has performed under loaded data using MATLAB simulator to reconstruct the image (Soleimani, 2006).

\section{DATA ACQUISITION}

EIT has flexibility to provide different schemes to utilize electrodes for current injection and in result voltage and current pattern is determined. According to literature review, lots of methods are there for data collection like adaptive, neighboring, cross, opposite methods. In this article, the neighboring method is used to support of the image algorithm for acquisition of data (Kumar et al., 2015). In this method, position of current is fixed to set of electrodes placed adjacent to each other for measuring voltage shown in figure 3 . Electrode 3 has been taken as reference electrode illustrated in figure 3. The applied current location electrodes have been chosen with respected to reference electrode and their combinations are represented as 3-4,3-5, 3-6, 3-7...3-16. On the change on current location, the reference will change and voltage measurement the depends potential difference from reference point to adjacent electrodes. If current referent point is 4 , then voltage measurement must be represented by $4-5,4-6,4-7,4-8,4-9 \ldots \ldots .4-1$. The distribution of electrodes has done as per above discussion by selection current reference electrodes and voltage measurement fully depends on the distances from reference electrode to adjacent current electrodes. The calculation of voltage measurement for among 


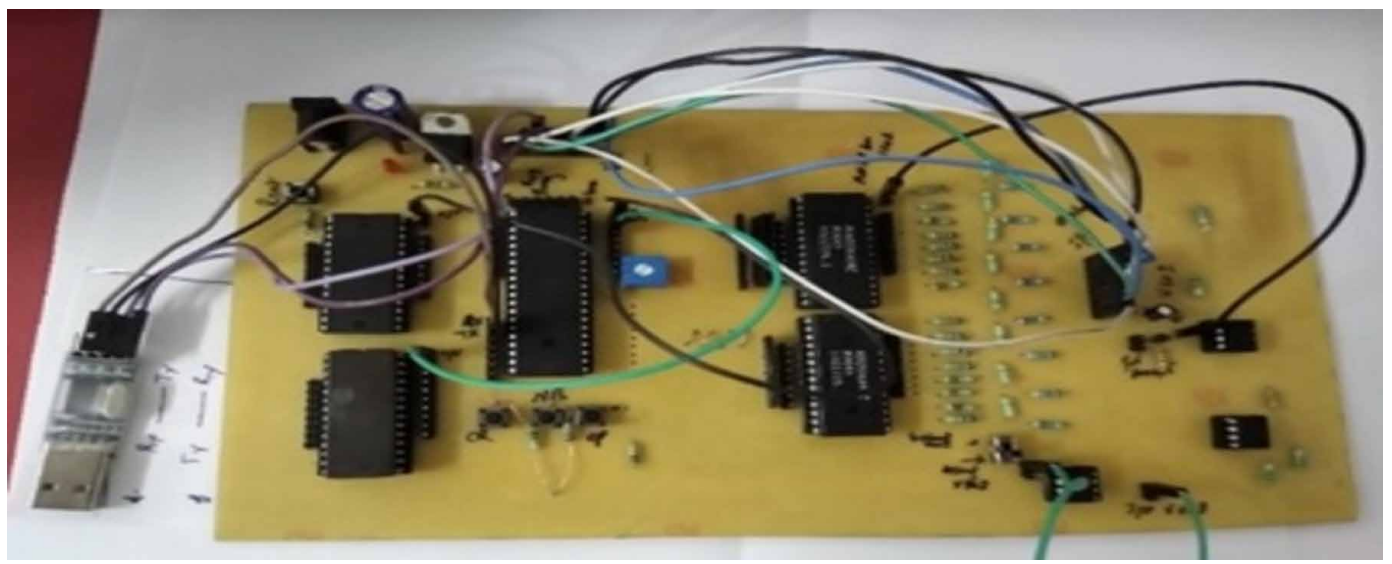

$\mathrm{N}$ electrodes with 3 reference electrodes may defined by $\mathrm{N}(\mathrm{N}-3)$. If total number of electrodes are 16 among 3 reference electrodes, then in the neighboring method, voltage measurements is concluded on the basis 208 measurements (Kumar et al., 2015).

\section{IMAGE RECONSTRUCTION ALGORITHM}

The algorithm for image reconstruction has play significant role in EIT. With the help of algorithm mathematics, resistivity images can be developed under the data collected from the phantoms. The mathematical algorithm initiated along conductivity of body to calculate potential by deployment of electrodes. EIT image reconstruction relate the internal distribution of conductivity for measurement of the boundary voltage of a physical model. The system defines all variable including the measured voltages, the injected current and the conductivity distribution and derived the physical model equation using Maxwell's equation (Hanke et al., 2011). To define the distribution of conductivity through the EIT inverse solution, the forward solution is needed. The solution of forward problem and inverse is to be needed to define to solution of EIT System. A FEM mesh of triangular nodes and elements are used to solve inverse problems and forward problems (Kilic et al., 1998). Conductivity of the uniform

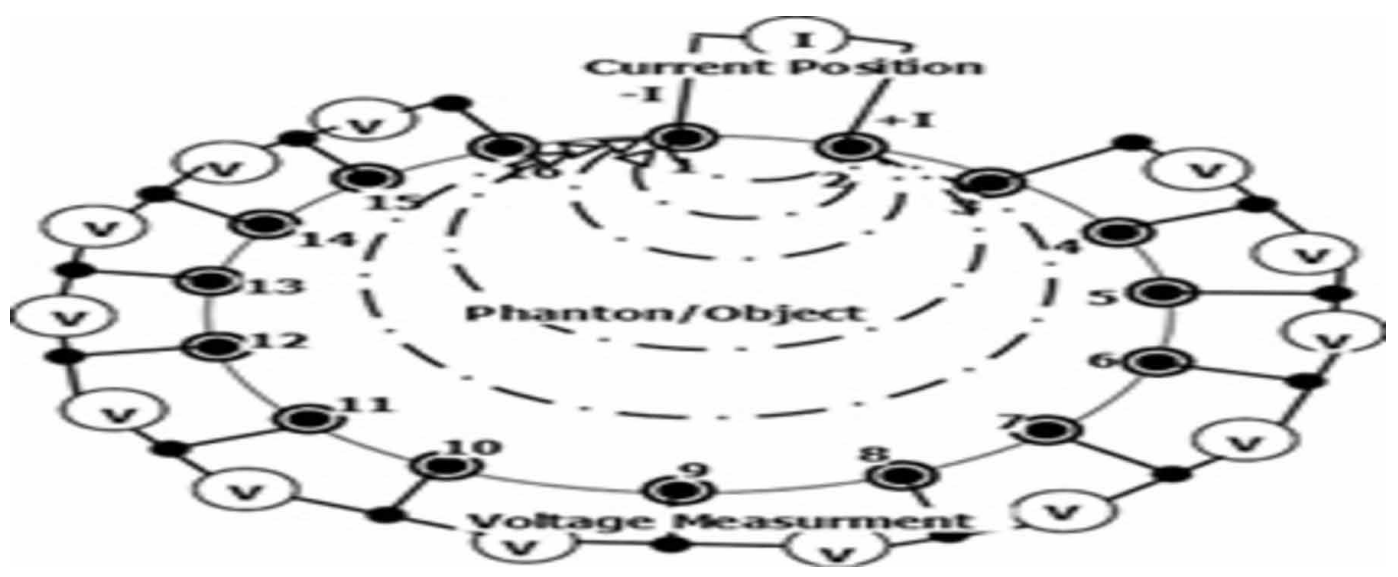


field, current injection pairs and amplitude of current are the variable that help to define solution of Forward problem. Whereas, boundary potential of the object is depending on impedance distribution and the process for calculation of the discussed variables is known as the inverse problem. In literature, various approaches have been discussed and developed to define the forward and inverse problem and Graphical User Interface window is used to define all process of the mathematical algorithms (MathWorks Inc, 2005). The program is more comfortable for users to provide solutions for said problems. It is reliable, intuitive controls, precise, predictable and understandable. So all parts of this algorithm are explained below step to step.

\subsection{EIT Physical Model}

In the experiment, boundary voltage variables are measured with respect to reference electrodes by applying on the alternating current of the order of a few mA from $1 \mathrm{kHz}$ to $100 \mathrm{kHz}$ frequency range to a electrodes subset (Kumar et al., 2008a). In this experiment, an array of electrodes with 16 or 32 are attached to the object. Further using different boundary conditions, the physical model for EIT is derived. Using Maxwell's equation, the mathematical model of the problem is derived (Cao et al., 2006).

$\tilde{\mathrm{N}} \times \mathrm{B}=-\Phi \mathrm{IB} / \mathrm{\Phi} \mathrm{t}$

$\tilde{\mathrm{N}} \times \mathrm{H}=\vartheta+\llbracket \Delta / \llbracket \tau$

Where $\tilde{\mathrm{N}}$ is the curl operator, $\mathbb{I} / \mathrm{I} \mathrm{t}$ partial derivative with respect to time, the Electric field abbreviated by $\mathrm{E}$, the magnetic field abbreviated by $\mathrm{H}$, the dielectric displacement abbreviated by $\mathrm{D}$, the electric current density abbreviated by $\mathrm{J}$ and the magnetic induction abbreviated by B. Current density for the linear isotropic medium is define by continuity equation (Lui et al., 2008). The divergence of $\mathbf{J}$ is written as

$\tilde{\mathrm{N}} . \vartheta=0$

The current density $\mathrm{J}$ and electric field intensity is equated by a proportionality constant known as conductivity of medium. The ohm's low is used to define the relationship between to discussed variable is given in below:

$\vartheta=\sigma . \mathrm{E}$

Here, $\sigma$ is known as the medium conductivity.

In EIT system typically low-frequency signals are used. Therefore, this problem is considered to be quasi or temporary. Static it means EIT mode of quasi-state approximation. Therefore, Maxwell's law for electrostatic forms is employed in EIT system (Lui et al., 2008). The relation between electrical field intensity and scalar electric potential is defined by Maxwell second equation for electrostatics (Cao et al., 2006), as given

$\mathrm{E}=-\tilde{\mathrm{N}} \phi$

Where $\phi$ is known as scalar electric ðeld potential.

Finally, the electric field reevaluated from the equation (3), (4), (5) and placing ohm's low (Cao et al., 2006) and the equation known as Laplace is given as 
The electric potential $\phi$ inside the body $\Omega$ is recovered by this equation. A suitable and reasonable model should be developed for connections of electrodes with in the object. The EIT decides the boundary conditions for Laplacian (Lui et al., 2008). The relation between current density at the boundary with the conductivity $\sigma$ and the electric potential is fellows as:

$\tilde{\mathrm{N}} \cdot(\sigma \tilde{\mathrm{N}} \phi)=0$

$$
\sigma \frac{\partial \phi}{\partial n} \| \partial A=J_{O}
$$

Where $\mathrm{J}_{0}$ represents current density on the boundary and IIA is known as the boundary of the object.

\subsection{Forward Problem}

The voltage density distribution for given conductivity, injecting current pairs and boundary condition is defined under the forward problem. The electric field distribution within a source-free crosssection is observed by EIT electrode model. FEM is based on the known vector of conductivity and transformation matrix and governed by Laplace partial differential equation of second order for the range of the frequencies and second order (Polydorides, 2002). The above discussion comes under the 'forward problem'. Henceforth, it is the procedure for measuring the boundary potential under impedance distribution of the object boundary (Soni, 2006). We are discussing various numerical methods that are used for solving the forward problem. The boundary element method (BEM), the finite difference method (FDM), the finite element method (FEM) and partial differential equations (PDE) are the methods used for solving the forward problem. Methods are also referred as analysis. Estimated solutions of partial differential equations/ integral equations are defined as FEM and used commonly for granting solutions of EIT problems efficiently (Saeedizadeh et al., 2012). Different boundary conditions/ geometric shapes can be defined by FEM (Soni, 2006). The process included with FEM shown in figure 4 in the form of flowchart.

By Mashing, we Formulate all equations and boundary conditions and Divide analysis domain into finite elements of the object, which is referred to as a node and the sides they are called nodal lines or planes. In Modelling Selecting the interpolation functions of the FEM, which is Formulation of finite element equations for elements. The surface projection technique uses the triangulated boundary as a means of image reconstructing the surface from the unstructured data sets. Most commonly used algorithm is Delaunay Triangulation. In step III and step IV, primarily referred for the solution of the global equation and all matrices related to FEM in EIT technique (Xu, 2005).

From the resultant Laplace equation 7, the restoring the electrical conductivity in EIT used to construct the physical model. Distributed Conductivity, injected current, low frequency current and electrical potential within object and measured voltage are defined by single equation. The mathematical details of material can be modeled under properties of electrodes. The uniqueness of EIT equation deals the complete electrode model of the electrodes under mixed boundary conditions (Saeedizadeh et al., 2012). The integral of current density across the surface of the object under each electrode is equal to the flow of current

$$
\int_{E} \sigma \frac{\partial \phi}{\partial \vec{n}} d \mathcal{S}=I_{l}, l=1,2,3, \ldots \ldots \ldots L
$$




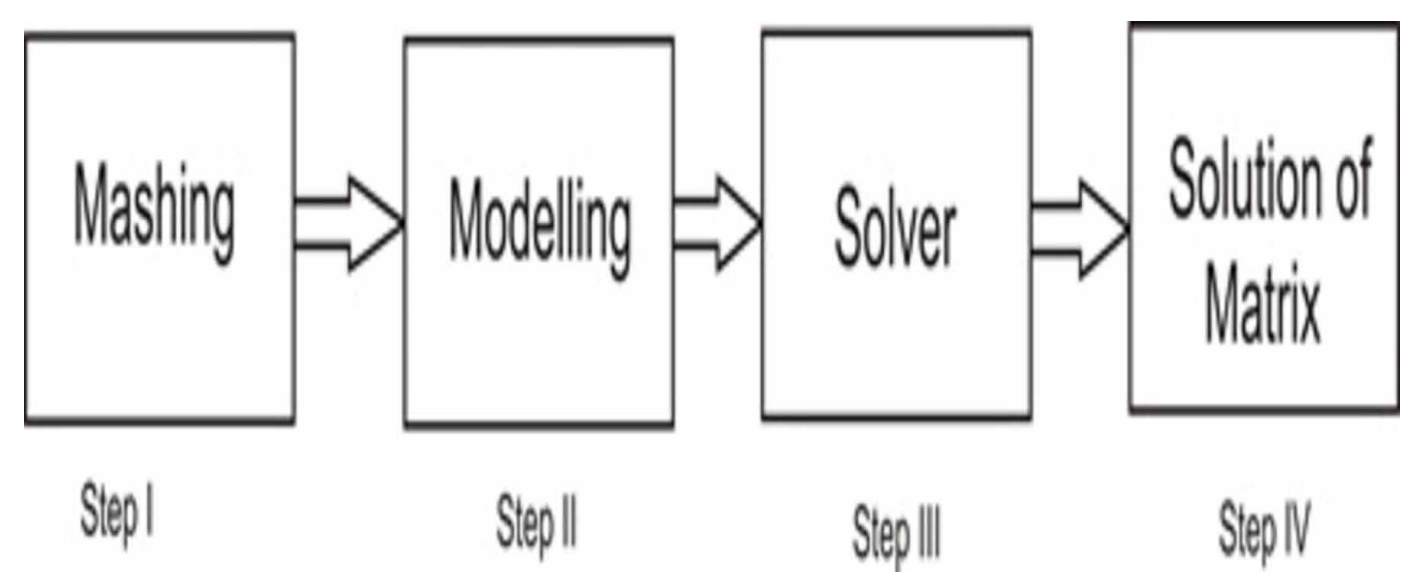

Where $\mathrm{I}_{1}$, current flow, $\mathrm{S}$ is the electrode surface, $\mathrm{L}$ number of electrodes.

The sum of the voltage on the subject surface and the voltage drop of the contact resistance is called measured voltage or potential of the electrode.

$\phi+Z_{i} \sigma \frac{\partial \phi}{\partial \vec{n}} d \mathcal{S}=V_{l}, l=1,2,3, \ldots \ldots \ldots L$

$\mathbf{V}_{1}$, Is the potential value, $l$ is the number of measuring electrodes and $\mathbf{Z}_{\mathbf{i}}$ is the contact resistance. When the law of conservation of charge satisfies the EIT model has a unique solution.

$\int_{\partial \Omega}^{\vec{j}}=0 \Leftrightarrow \sum_{i=1}^{L} I_{l}=0$

And the selection of ground is made based on equation 12

$$
\int_{\partial \Omega} \phi=0 \Leftrightarrow \sum_{i=1}^{L} V_{l}=0
$$

To solve the elliptic problem, the finite element method is mostly used. Simplexes set of the finite elements are used to approximate the spatial area in the finite element method. The simplexes become triangle in the two-dimensional space. Thus in the two-dimensional case, $\mathrm{K}$ and $\mathrm{N}$ is the number of triangles. A set of the simplex is called FEM mesh (Polydorides, 2002). The essential functions are responsible for the potential approximation that is equal to unity in $i_{\text {th }}$ mode and equal to zero at all other nodes.

$$
\phi(\vec{r})=\sum_{i=1}^{N} \phi_{i} \omega_{i}(\vec{r})
$$

$\boldsymbol{\phi}_{\mathrm{i}}, \boldsymbol{\omega}_{\mathrm{i}}$ is discretized potential, basis functions respectively.

Therefore the finite element representation of the equation EIT (Polydorides, 2002). 


$$
\begin{array}{cc}
\mathrm{A}_{\mathrm{M}}+\mathrm{A}_{\mathrm{Z}} & \mathrm{A}_{\mathrm{W}} \vec{\phi} \\
\mathrm{A}_{\mathrm{W}}^{\mathrm{T}} & \mathrm{A}_{\mathrm{D}} \overrightarrow{\mathrm{V}}
\end{array}=\begin{aligned}
& \overrightarrow{0} \\
& \overrightarrow{\mathrm{I}}
\end{aligned}
$$

$\vec{V}, \vec{\phi}=\left(\phi_{1}, \ldots . . \phi_{n}\right)^{T}, \vec{I}$ is vector a of the potentials, vector a containing the current, vector that represents a discrete approximation respectively.

\subsection{Inverse Problem}

A forward model is developed to calculate the "inverse problem" under EIT and the voltage referred injected current for a known conductivity distribution is calculated. This model could be used to compare the measured data voltage in an iterative procedure for evaluating conductivity and also to predict the observed voltage given the estimated conductivity (Vauhkonen, 1997). EIT method is extremely effected by the "inverse problem" due to non-linearity (Janicke \& Kost, 1996). Which has capacity to rebuilt the distribution of conductivity under evaluation of boundary potential. Which is also known as process of image recovering from the boundary data signal. "Jacobian matrix, Tikhonov regularization method, Gauss-Newton Algorithm”, etc. are some algorithms are defined inverse problem. so, here we used the Jacobean mathematical method for the inverse problem (Seok, 2014). The Jacobian matrix is constructed using the next field method with the help of internal electric field which is calculated by the forward model. The Jacobian matrix is designed to understand the known voltage under unknown conductivity in the inverse problem (Polydorides, 2002).

In EIT problem the measurement of the background conductivity distribution consists of the significant change in the result as compared with the changes in the result concerning the input of medium, conductivity. Static and dynamic are the two component of the forward problem in the equation, and the dynamic component is considered to be a linear function of the conductivity change (Olmos, 2012).

$$
v_{h}+\Delta v=F\left(\sigma_{h}\right)+\mathbf{J} \Delta \sigma
$$

In this equation, $v_{h}, \ddot{\mathrm{A}} v, F\left(\sigma_{h}\right), \ddot{\mathrm{A}} \sigma, J$ is the voltage measurements from a homogeneous medium, change in measurements, function of the homogeneous conductivity distribution, change in conductivity distribution and Jacobian matrix which describes this linear relation respectively. This linear approximation is valid for a limited range. Thus for a small change in conductivity i.e. $\Delta \sigma$ the inverse problem is linearly modelled and for large conductivity change a nonlinear model is more accurate in which the inverse problem is solved by iterative approaches

$$
z=J x+n
$$

Here $\mathrm{z}, x, n J$ is the deference measurement $(\Delta \mathrm{v})$, the conductivity change $(\Delta \sigma)$, the noise model that appears in the measurements which is considered an uncorrelated additive white Gaussian Noise (AWGN) and the Jacobian matrix respectively. Jacobian matrix $(\mathrm{J})$ is the matrix of partial derivatives of voltage with respect to conductivity parameters. ' $\mathrm{J}$ ' is also called sensitivity matrix and its rows are called sensitivity map in the industrial and medical EIT literature. The system matrix could be used to drive sensitivity matrix by the equation 17 . 


$$
J=T \frac{\partial}{\partial \sigma} S^{-1}(\sigma) I=T S^{-1}(\sigma) \frac{\partial}{\partial \sigma} S(\sigma) S^{-1}(\sigma) I
$$

Where $T, S, I, S^{-1}(\sigma) I$ is an operator for extracting measurements from the nodal voltages, the systems matrix developed in the forward model, injected current and the voltage of the nodes which is the solution of the forward problem respectively.

In a model Jacobian is a $K \times b$ matrix consist of $\mathrm{K}$ elements and boundary measurements. The Jacobian is a column by column matrix in which column represents an element. Hence $v_{i}$ measured between electrode pairs, the $\mathrm{i}^{\text {th }}$ column represents the effect of the conductivity change in the $\mathrm{j}^{\text {th }}$ element on the measured voltage (Woo et al., 1993). The conductivity change in an element to a measured voltage is related by each element of $\mathrm{J}$ at a background conductivity $\sigma_{0}$ as

$$
J_{i j}=\frac{\partial v_{i}}{\partial \sigma_{j}} \| \sigma_{0}
$$

Here, $v_{-} i$ is the $j_{\text {th }}$ measurement voltage. The sensitivity matrix ' $J$ ' is a function of the current injection pattern, the finite element model and the background conductivity for each of the elements a background conductivity is supposed to be homogenous i.e. $\sigma_{-} 0=1$. Since Jacobian matrix (J) is not a square matrix, therefore to solve the linearized inverse problem is not straightforward, unlike the forward problem. The least square solution is one approach which is used to minimize and the solution should be calculated by this approach as

$$
\hat{x}=\left(J^{T} J\right)^{-1} J^{T} z
$$

Therefore the matrix $\mathrm{J}^{\wedge} \mathrm{T} \mathrm{J}$ is the rank deficient and cannot be inverted because the problem in equation 19 is ill-posed and there are the unknown conductivities than the known measurements. A small change in the input data results in a significant change in the output in ill-conditioned problems, hence a small error in the data or any noise signal can be corrupted entirely its unique and existing solution (Olmos, 2012). The generalized regularization formula is used to reconstruct the conductivity $\hat{x}$.

$$
\hat{x}=\left(J^{T} J+\lambda^{2} R^{T} R\right)^{-1} J^{T} z=B z
$$

Here this equation is consists of a regularization parameter or 'hyper-parameter' and regularization matrix. The regularization problem is reduced to the Tikhonov regularization by the most straightforward form the regularization matrix, i.e., is the identity matrix I or R=I, the most common regularization matrix is the identity matrix and the matrixes corresponding to the first and second difference operators are employed in EIT. Therefore the primary function of the regularization is to solve the following minimization problem instead of the least square minimization (Jehl, 2014).

$$
\hat{x}=\arg \min _{x} J x-z^{2}+\lambda^{2} R_{x}^{2}
$$


Where $\lambda^{2} R_{r}^{2}$ expresses some prior information about the conductivity and reduces the illconditioning of the problem by adding more constraints.

\subsection{Graphical User Interface}

A GUI is a user friendly interface window. A good GUI window can be made programs more convenient to use. A basic operation of GUI window flowchart is shown below in figure 5 .

According to the flowchart, every item of GUI is a graphical component include pictorial controls that include edit boxes, push buttons, sliders, lists, etc. The all components of a GUI must be arranged within a GUI window, that can be design with the figure and can be operated any combination of components. There is some way to act, like a button type information. After that, directly respond the MATLAB program of the event, according to the button click.

GUI is user friendly interface allow to design an image reconstruction algorithm with defined components. It provides an easier scenario in the field of programming. In MATLAB, modification

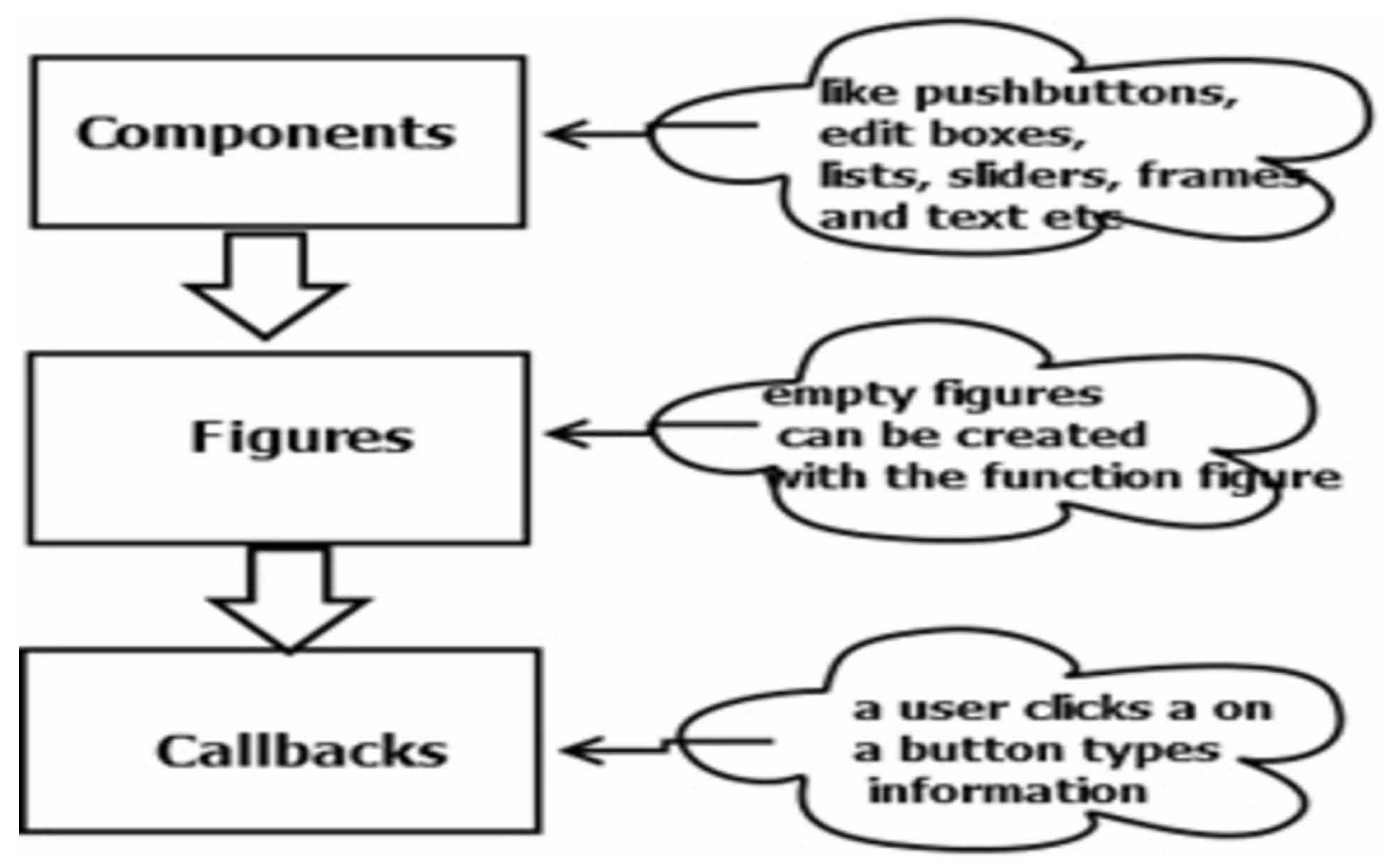

in coding or function is very easy with the help of an m file that provide GUI platform. Data extracted from EIT system is very significant to understand impedance analysis. GUI software is designed to understand behavior of data provided by EIT system. All functions of EIT system is controlled by GUI controls. A flowchart of GUI based algorithm has been depicted in figure 6 .

GUI functions facilitate users for selection of data acquisition methods by choosing number of electrodes. The image obtained from data provided under FEM in EIT system, GUI window has following implemented functionality: (a) availability of electrodes selection option; (b) Different data acquisition methods availability under electrical impedance-based technique; (c) option for selecting the FEM elements that provide the variable image resolutions; (d) option for selecting data mode may be used offline as well as online mode; (e) option for image reconstruction under FEM model under acquired voltage data of EIT system. GUI is developed and illustrated in Figure 7. 


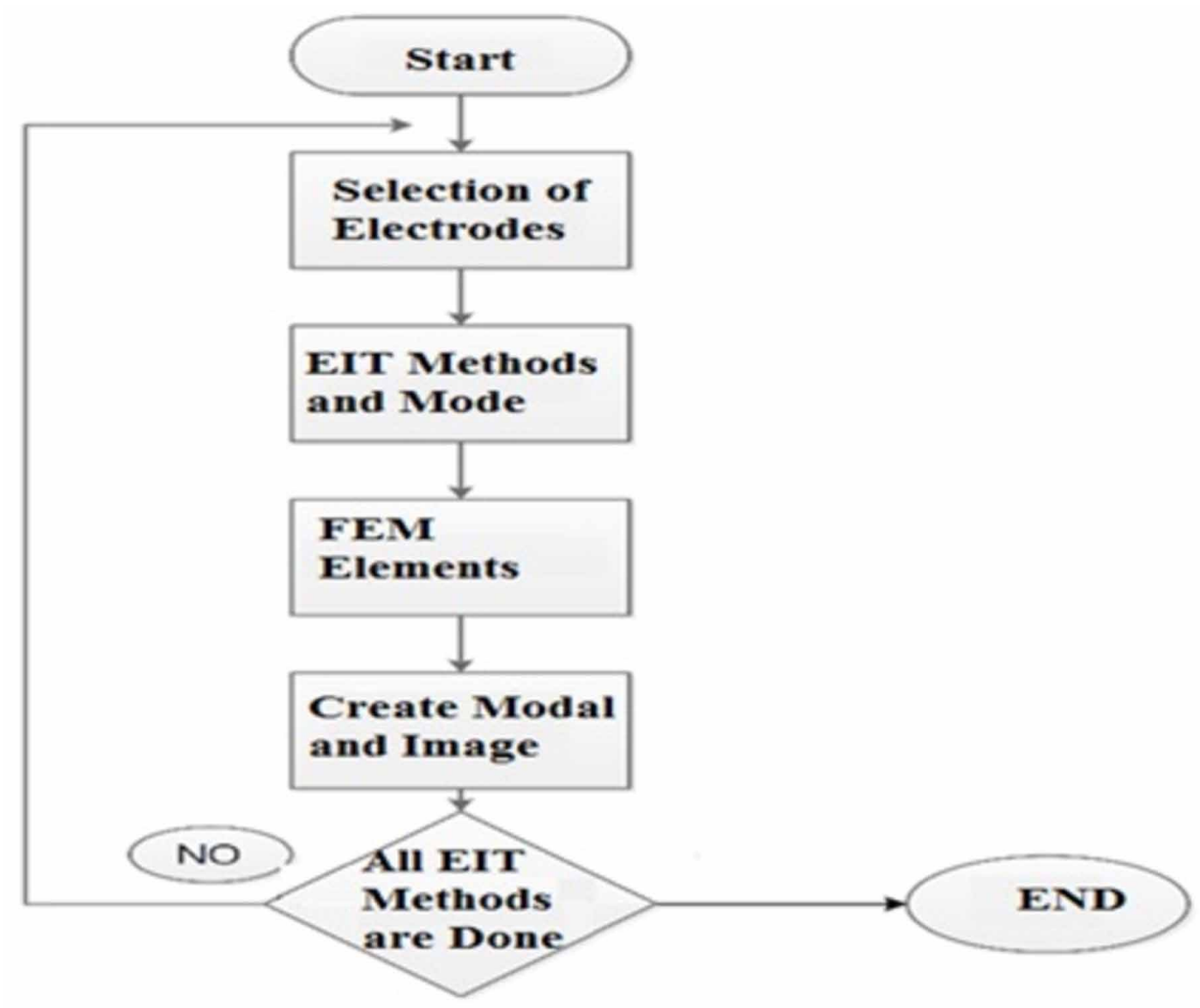

\section{EXPERIMENTAL WORK}

By developing the practical system for electrical impedance tomography, many research groups use the different measuring system for image reconstruction algorithms. For comparison between reconstruction algorithms, a numerical model for simulating EIT measurements are very useful which is easily exchanged between centers and essentially free-form noise. To design a physical phantom which is consistent and stable in construction for comparing complete EIT system and can be distributed to all the concerned centers. GUI is allowed to measure resistivity and resolution at any point within the phantom as per known amount of electrical conductivity. The transparent electrolytes used in phantom are different and create error due to change in conductivity by the evaporation of the water. In the phantom creation, the first role is to search a material suitable for an EIT imaging. This technique is developed for object scanning using electrical impedance tomography using a reliable quantitative data processing protocol.

The goal of the experiment is gather values of voltage from different types of the phantom. The reconstruction of image is happened under support of the array of the sensor and geometry of practical phantom. Due to assumption of restricted current conduction by EIT, the model is not valid for a real volume conductor. The phantom is designed on the basis of parameters like electrode geometry, electrode materials, phantom dimension to reconstructed image accuracy and resolution. Images are used for EIT. EIT provides flexibility to examine response of phantom on various electrode 
Figure 7. Graphical User Interface

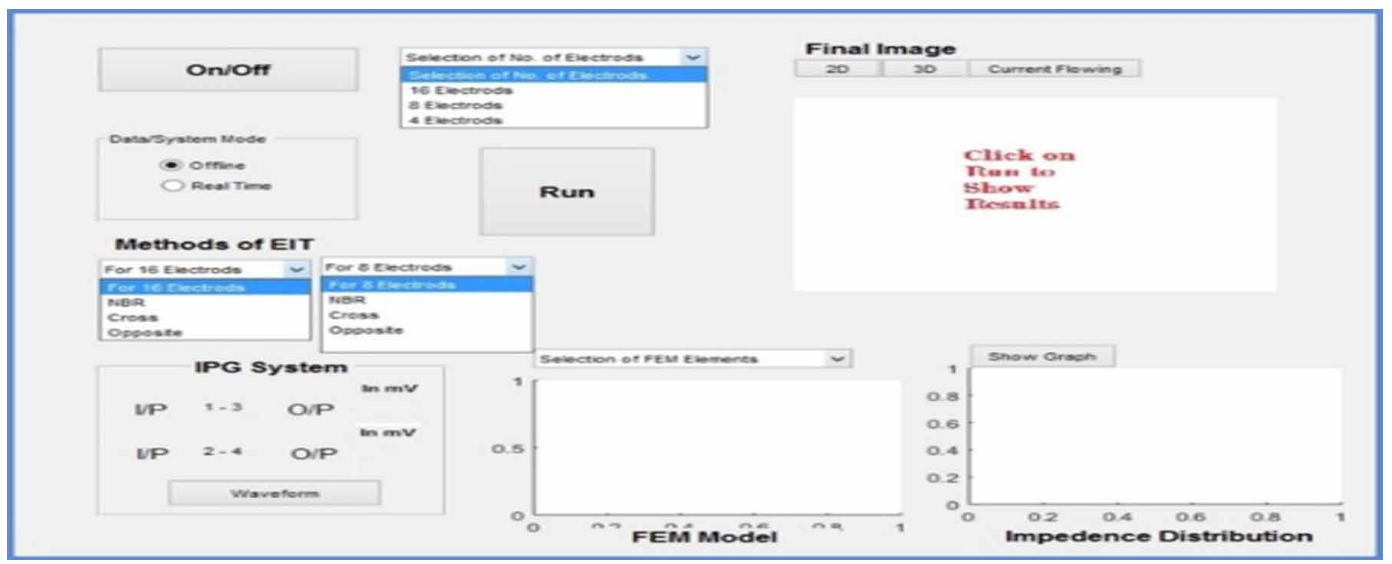

Figure 8. Experimental Setup for Papaya Phantom
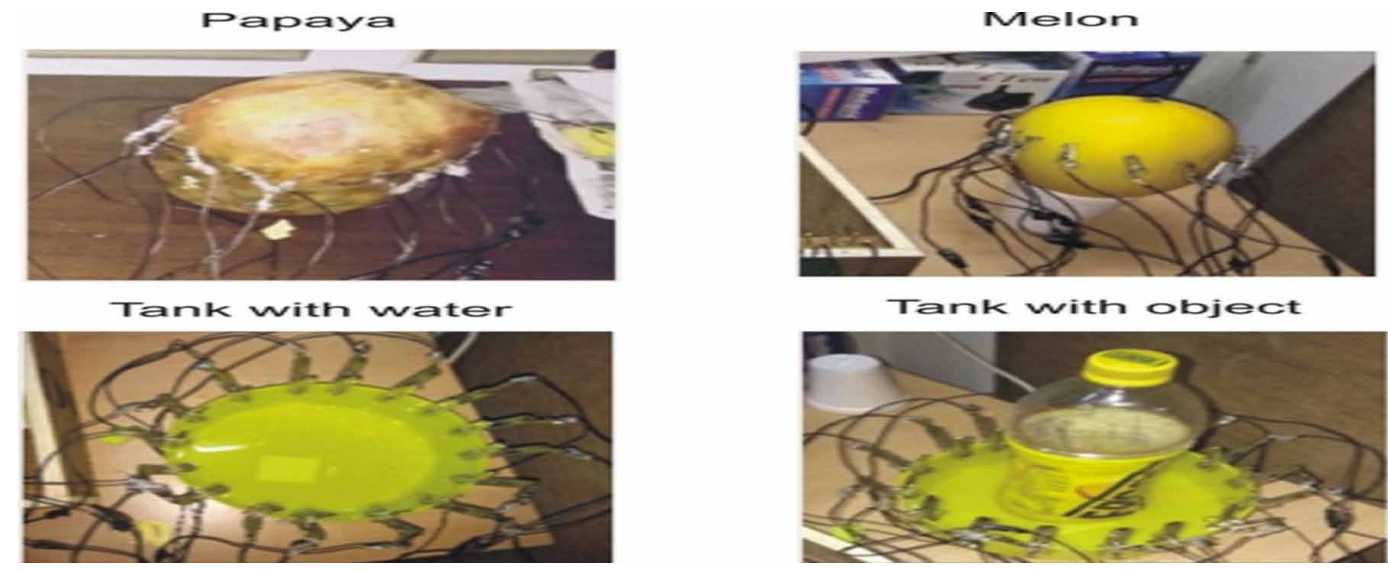

location. To remove the measurement error for the better quality of image an EIT phantom and simple experimentation are developed, as shown in figure 8.

So for this, we have taken some phantoms like Papaya, melon, plastic tank with saline water and tank with object that is analogous to the human stomach inserted electrode over its circumference and then applied current using function generator then calculated the potential from other remaining electrode using the neighboring method.

\section{RESULTS}

To obtain the EIT imaging the voltage measuring and current driving method has been used. To inject current between a pair of adjacent electrodes an adjacent driving and measuring pattern is employed and remaining electrode pairs are used to measure the potential difference. One set of measurement is completed with 16 electrodes used as driving electrodes when placed within a single plane. The impedance distribution curves measured for all 16 electrodes as shown in the figure 9 and figure 10, an applied signal current $1 \mathrm{~mA}$ at $50 \mathrm{KHz}$ frequency is used for measurements. The GUI window consists of 2D image different types of mesh models and impedance distribution graph with simulation 
Figure 9. Final Result for used phantoms according to experimental setup

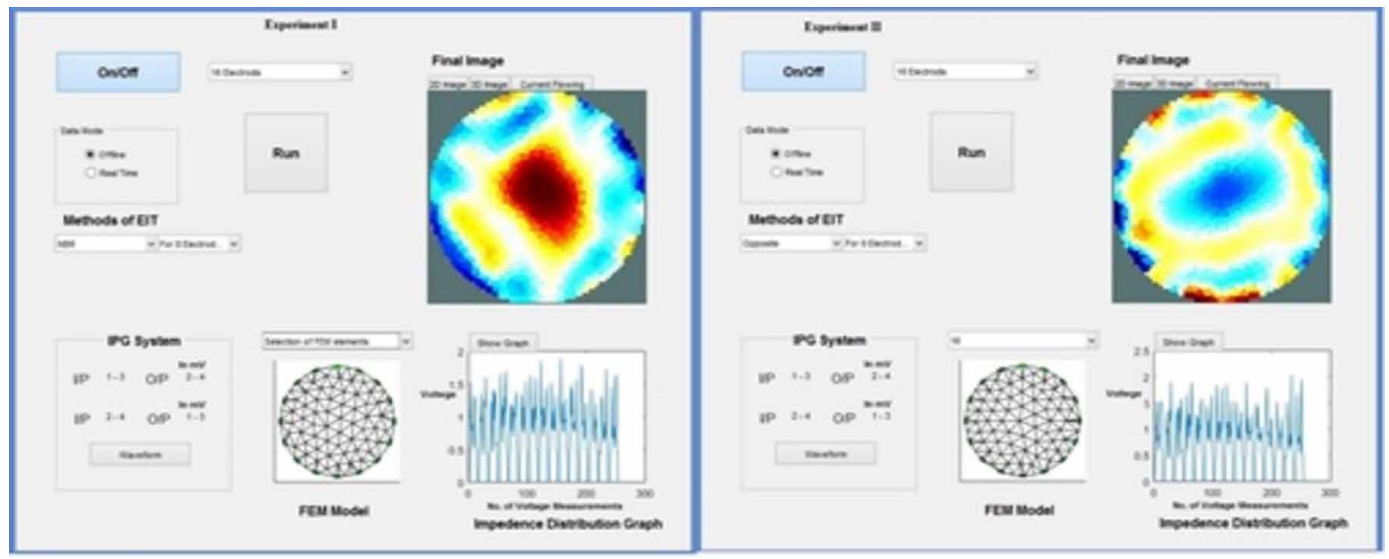

result according to action by the EIT system. The voltage on the electrodes and potential inside the domain is given by the forward solution.

The mesh is used to compute the potential distribution. The location of the electrodes is identified. The 2D image is the final stage for simulation results. The number of finite elements decided it. The image becomes more evident when the number of finite elements increases. A platform for fast prototyping of different reconstructions schemes in EIT is provided by the existing set of MATLAB functions. The results can be displayed and analyzed quickly with code medication which is relatively straight forward.

\section{DISCUSSION}

In above Figures shown some reconstructed images in form of GUI. The GUI is containing a 2D image, a FEM model, Impedance distribution graph, data acquisition method, and electrodes pattern of the phantoms obtained from the performed experiments from the proposed system is illustrated. These images were reconstructed using finite element method (FEM) and Jacobean matrix algorithm through the GUI on MATLAB platform. The comparable results have shown from the obtained $2 \mathrm{D}$ images according to the obtained data through the proposed EIT system. According to 16 or 8 electrodes combination. In above figures, clearly defines the internal structure of the phantoms, which based on internal impedance distribution. The better image is obtained through the 16 electrodes combination as compared to 8 electrode configuration. Clear and distinct image were obtained after reconstruction based on conductivity distribution of the phantom. It is demonstrated that proposed EIT system is capable for the industrial as well as biomedical applications.

\section{CONCLUSION}

EIT is a portable, non-invasive and low-cost imaging method. The bio-impedance has wide applications with respect to clinical and nonclinical, due to better ability in imaging analysis of the phantoms. It is based on the fact that different tissues of living cells of the body will present different conductivity distribution under apart circumference points. The output surface voltage is measured by applying input current to reconstruct the image according to internal conductivity distribution. According to final results, the model gives the 2D image, FEM mesh model, impedance distribution graph with respect to the internal potential distribution. Thus, we conclude the proposed reconstruction algorithms to improve for better image resolution. The future improvement possibility is that in the 
Figure 10. Final Result for used phantoms according to experimental setup

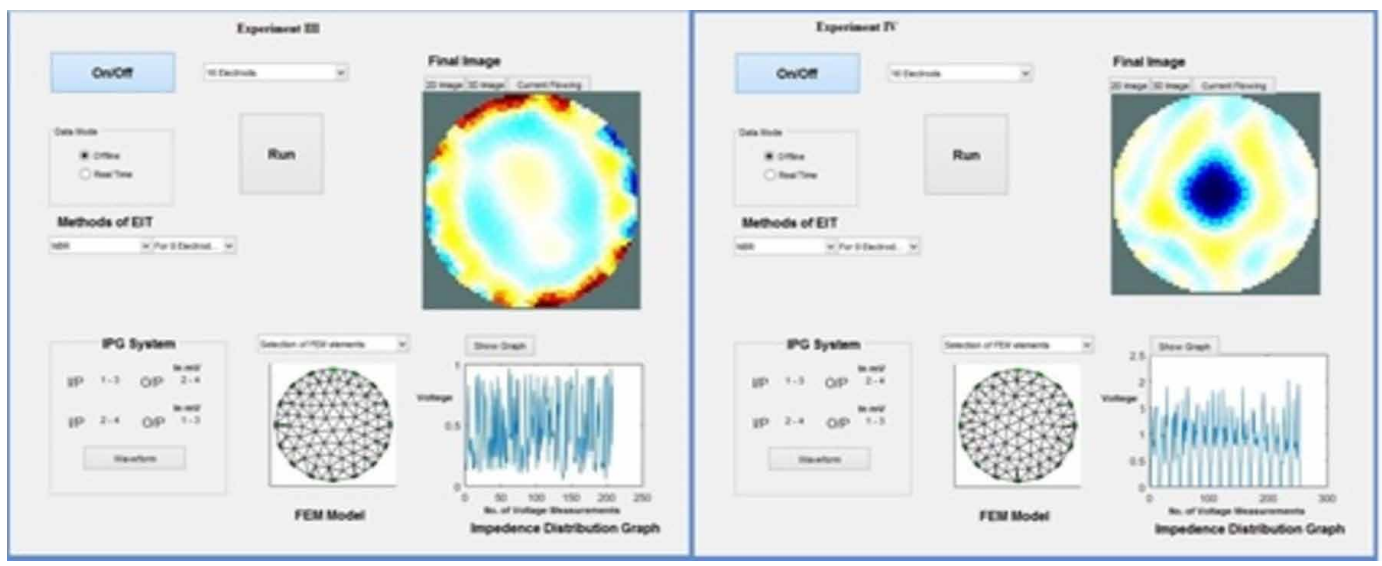

3D image and better resolution and analysis of image according to the numerical method of the image reconstruction algorithm. It is demonstrated that the proposed EIT system is capable for the industrial as well as biomedical applications.

\section{REFERENCES}

Borsic, A., \& Bayford, R. (2010). Forward Solving in Electrical Impedance Tomography with Algebraic Multigrid Wavelet Based Preconditioners. Journal of Physics: Conference Series, 224, 12-53. doi:10.1088/1742$6596 / 224 / 1 / 012053$

Cao, Z., Wang, H., \& Dong, F. (2006). The Study of a 2D Model and Image Reconstruction Algorithms Based on EIT System. 2006 IEEE Instrumentation and Measurement Technology Conference Proceedings, 2323-2326. doi:10.1109/IMTC.2006.328613 
Hanke, M., Harrach, B., \& Hyvönen, N. (2011). Justification of Point Electrode Models in Electrical Impedance Tomography. Mathematical Models and Methods in Applied Sciences, 21(06), 1395-1413. doi:10.1142/ S0218202511005362

Hinz, J., Hahn, G., \& Quintel, M. (2005). Electrical Impedance Tomography. Der Anaesthesist, 57(1), 61-69. doi:10.1007/s00101-007-1273-y PMID:17934702

Holder, D. S. (2005). Electrical impedance tomography Methods, History and Applications. Institute of Physics Publishing Bristol and Philadelphia.

Janicke, L., \& Kost, A. (1996). Error Estimation and Adaptive Mesh Generation in the 2D and 3D Finite Element Method. IEEE Transactions on Magnetics, 32(3), 1334-1337. doi:10.1109/20.497492

Jehl. (2014). A Fast Parallel Solver for the Forward Problem in Electrical Impedance Tomography. IEEE Transactions on Bio-Medical Engineering.

Khalighi, M. (2012). Practical Design of Low-Cost Instrumentation for Industrial Electrical Impedance Tomography (EIT). 2012 IEEE I2MTC - International Instrumentation and Measurement Technology Conference, Proceedings, 1259-1263. doi:10.1109/I2MTC.2012.6229173

Kilic, Beyhan, \& Kork. (1998). A Finite element method based neural network technique for image reconstruction in electrical impedance imaging. International Biomedical Engineering Days, 100-102.

Kumar, Anand, \& Sengupta. (2008a). Development of a Non - Invasive Point of Care Diagnostic Tool for Fetal Monitoring Using Electrical Impedance Based Approach. IEEE Point-of-Care Healthcare Technologies, 16-18.

Kumar, S., Anand, S., \& Sengupta, A. (2008b). FETO-Maternal Monitoring Using Electrical Impedance Tomography Method. Elsevier.

Kumar, Kumar, \& Sengupta. (2015). A Review : Electrical Impedance Tomography System and Its Application. Journal of Control and Instrumentation, 7(2), 14-22.

Lui, , Lili, \&, \& Hull, . (2008). Analysis of the electrical impedance tomography algorithm based on finite element method and tikhonov. International Conference on Wavelet Analysis and Pattern Recognition, 13-16.

MathWorks Inc. (2005). Creating Graphical User Interfaces. MATLAB User Guide, x, 10-20.

Mengxing, T., Xiuzhen, D., Mingxin, Q., Feng, F., Xuetao, S., \& Fusheng, Y. (1998). Electrical Impedance Tomography Reconstruction Algorithm Based on General Inversion Theory and Finite Element Method. Medical \& Biological Engineering \& Computing, 36(4), 395-398. doi:10.1007/BF02523205 PMID:10198520

Olmos, M. (2012). A Reconstruction Method for Electrical Capacitance Tomography Based on Image Fusion Techniques. Digital Signal Processing, 22(6), 885-893. doi:10.1016/j.dsp.2012.07.002

Polydorides, N. (2002). Image Reconstruction Algorithms for Soft-Field Tomography (Ph.D. thesis). Polydorides Manchester.

Qiu, C., Hoyle, B. S., \& Podd, F. J. W. (2007). Engineering and application of a dual-modality process tomography system. Flow Measurement and Instrumentation, 18(5-6), 247-254. doi:10.1016/j.flowmeasinst.2007.07.008

Saeedizadeh, N., Kermani, S., \& Rabbani, H. (2012). A Comparison between the hp-version of Finite Element Method with ... Academic Press.

Seok, B. (2014). Image Reconstruction Using Voltage-Current System in Electrical Impedance Tomography. Nuclear Engineering and Design, 278, 134-140. doi:10.1016/j.nucengdes.2014.07.023

Soleimani, M. (2006). Electrical Impedance Tomography System: An Open Access Circuit Design. Biomedical Engineering Online, 5(1), 28. doi:10.1186/1475-925X-5-28 PMID:16672061

Soni. (2006). Finite Element Implementation of Maxwell's Equations for Image Reconstruction in Electrical Impedance Tomography. IEEE Transactions on Medical Imaging, 55-61.

Vauhkonen, M. (1997). Electrical impedance tomography and prior information (Ph.D. thesis). Kuopio University Publications. 
Woo, E. J., Hua, P., Webster, J. G., \& Tompkins, W. J. (1993). A Robust Image Reconstruction Algorithm and Its Parallel Implementation in Electrical Impedance Tomography. IEEE Transactions on Medical Imaging, 12(2), 137-146. doi:10.1109/42.232242 PMID:18218401

Xu, G. X. G. (2005). 3-D Electrical Impedance Tomography Forward Problem with Finite Element Method. IEEE Transactions on Magnetics, 1832-1835. 